\title{
An Uplink Hybrid Multiple Access Scheme for Wireless Communication Systems
}

\author{
Kan Zheng, Yuyu Wang, Hang Long, Meiying Wei, Wenbo Wang \\ Wireless Signal Processing and Network Lab \\ Key laboratory of Universal Wireless Communication, Ministry of Education \\ Beijing University of Posts\&Telecommunications \\ Beijing, China, 100088 \\ Email: kzheng@ieee.org
}

\begin{abstract}
Both orthogonal frequency division multiplexing (OFDM) and single-carrier (SC) with frequency division multiple access (FDMA) have been regarded as a possible solution for improving the performance of future generation wireless communication systems. OFDM can better exploit the frequency diversity gain while SC-FDMA is less sensitivity to non-linearity of amplifier with lower peak-to-average power ratio (PAPR). In this paper, a new hybrid multiple access scheme is proposed in order to combine the advantages of both OFDM and SCFDMA schemes, while the effects of both schemes on the realistic system assumptions of the practical high power amplifier (HPA) model and adaptive modulation/coding (AMC) technique are considered. Our simulation results have demonstrated the effectiveness of the proposed scheme and the conclusion is given.
\end{abstract}

\section{INTRODUCTION}

Orthogonal frequency division multiplexing (OFDM) has been chosen as the multiple access method in the third generation (3G) long-term evolution (LTE) and its further advancement because it can provide high data rates and spectrum efficiency [1] [2]. However, the large Peak-to-Average Power Ratio (PAPR) of OFDM signals decreases the power efficiency of transmitter front-end and causes the non-linear interference. Compared with the downlink transmission, such problem becomes more serious for the uplink transmission because usually the linear region of amplifier at the battery driven mobile user is much smaller.

So, there has been much attention on another broadband technique, namely single-carrier (SC) with Frequency Division Multiple Access (FDMA), also called as DFT-spread OFDM (DFTS-OFDM). SC-FDMA is a transmission scheme that has several good properties. Similar to OFDM, the inter-symbolInterference (ISI) in SC-FDMA systems can be eliminated completely using redundant cyclic prefix. The equalization is carried out in the frequency domain instead of in the time domain, which has less implementation complexity in the rich multipath environments. Compared with OFDM, one prominent advantage over OFDM is that the SC-FDMA signal has lower PAPR. Therefore, it has been selected as the uplink multiple access scheme for 3G LTE systems [1].

Most of existing literatures only evaluate the impacts of nonlinearity on error rate performance with the given modulation in OFDM systems [3] [4]. In addition, the performances

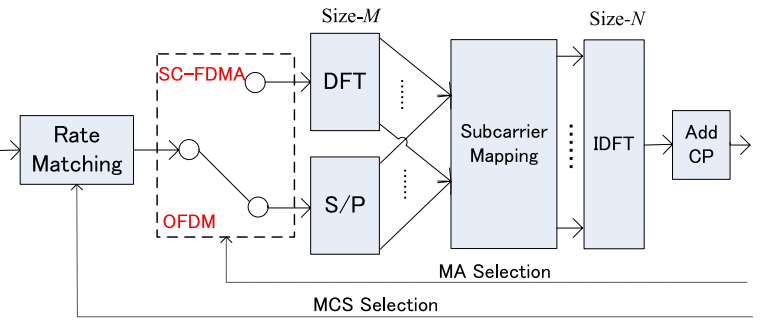

Fig. 1. Diagram of generalized multi-carrier transmitter in the uplink.

between OFDM and single-carrier with cyclic prefix (CP) are compared in term of error rate while considering the nonlinearity effects of amplifier in [5]. Moreover, the performances of these two uplink multiple access schemes including OFDM and SC-FDMA with the practical high power amplifier (HPA) model are compared in term of data throughput, where the adaptive modulation and coding (AMC) technique with slow power control is applied in [6].

In order to combine the advantages of both schemes including OFDM and SC-FDMA, a hybrid multiple access scheme is proposed through monitoring the varying error probability performance. The effects of both schemes on the realistic system assumptions of the practical high power amplifier (HPA) model and adaptive modulation/coding (AMC) technique are considered. The deployment of this new scheme is expected to reduce the size and power requirements of mobile device compared to conventional schemes, enabling the downsizing of back-up batteries, and thereby making a major contribution to reducing the costs of constructing the broadband wireless networks.

This paper is organized as follows. Section II gives the brief overview of a system model. The new multiple access scheme is described in Section III. Then, Section IV gives the simulation results. Finally, Section V gives the conclusion. 


\section{System OVERVIEW}

As shown in Fig.1, the generalized multi-carrier transmitter in the uplink is considered in this paper. Most of the linearly modulated signal types can be generated by this common way.

\section{A. Generalized Multi-carrier Signal}

A data-modulated symbol vector $\mathbf{d}=$ $\left[d_{0}, d_{1}, \cdots, d_{M-1}\right]^{T} \in \mathbb{C}^{M \times 1}$ is transformed by multiplying with the matrix $\mathbf{T} \in \mathbb{C}^{N \times M}$ before being fed to $N$-point inverse Discrete Fourier Transform(IDFT) to form the generalized multi-carrier signal as

$$
\mathbf{s}=\mathbf{F}_{N}^{H} \mathbf{T} \mathbf{d}=\left[s_{0}, s_{1}, \cdots, s_{N-1}\right]^{T} \in \mathbb{C}^{N \times 1},
$$

where $\mathbf{F}_{N}$ is an $N \times N$ DFT matrix, defined by

$$
\left\{\mathbf{F}_{N}\right\}_{m, n}=\frac{1}{\sqrt{N}} e^{-j 2 \pi n m / N}, \forall m, n \in\{0,1, \cdots, N-1\} .
$$

The OFDM signal is given by choosing $\mathbf{T}$ as a matrix in which all the diagonal elements are ones and others zeros. Then, by inserting rows of zeroes in this identity matrix we can get OFDMA signal, in which data-bearing subcarriers are selected based on diversity or traffic considerations. Also, the signal of localized SC-FDMA can be formed, in which $\mathbf{T}$ contains a DFT matrix of size $M$, i.e.

$$
\mathbf{T}=\left[\begin{array}{l}
\mathbf{F}_{M} \\
\mathbf{0}
\end{array}\right],
$$

where $\mathbf{F}_{M}$ is an $M \times M$ DFT matrix, whose element at the $m$ th row and $n$th column is $(1 / \sqrt{M}) e^{-j 2 \pi n m / M}$ for $0 \leq m, n \leq M-1$, and 0 is an $(N-M) \times M$ matrix of zeroes.

Subsequently, a cyclic prefix (CP) is added to avoid intersymbol-interference (ISI) and inter-carrier-interference (ICI) before the signal is transmitted over a multipath fading channel.

\section{B. HPA Model and its effects}

To simplify the discussion, only memoryless non-linearity of HPA is assumed in this paper. The output vector of HPA can be expressed as $\hat{\mathbf{s}}=\left[\hat{s}_{0}, \hat{s}_{1}, \cdots, \hat{s}_{N-1}\right]^{T} \in \mathbb{C}^{N \times 1}$ with

$$
\hat{s}_{n}=\left|s_{n}\right| G\left(\left|s_{n}\right|\right), \forall n \in\{0,1, \cdots, N-1\},
$$

where

$$
G\left(\left|s_{n}\right|\right)=\frac{A\left(\left|s_{n}\right|\right)}{\left|s_{n}\right|} e^{j \Phi\left(\left|s_{n}\right|\right)} .
$$

The function $A(\cdot)$ and $\Phi(\cdot)$ denote the AM/AM and AM/PM conversion characteristics of HPA, respectively. According to the Rapp model for the solid state power amplifier (SSPA) [7], the characteristics of HPA are given by

$$
A(r)=\frac{\nu r}{\left[1+\left(\nu r / A_{\text {sat }}\right)^{2 p}\right]^{1 / 2 p}}, \Phi(r) \simeq 0,
$$

where $A_{\text {sat }}$ is the saturated output level of the amplifier, $\nu$ is the small-signal gain and $p$ is an integer parameter that controls the smoothness of the transition from the linear region to the saturation region. The AM/AM curves approach the ideal limiter model when $p \rightarrow \infty$.
TABLE I

APPROXIMATE COEFFICIENTS OF MCS MODES AND SNR BOUNDARY

\begin{tabular}{|c|c|c|c|c|c|}
\hline & MCS1 & MCS2 & MCS3 & MCS4 & MCS5 \\
\hline Modulation & QPSK & 16QAM & 16QAM & 64QAM & 64QAM \\
\hline Coding Rate & $\frac{602}{1024}$ & $\frac{490}{1024}$ & $\frac{616}{1024}$ & $\frac{466}{1024}$ & $\frac{666}{1024}$ \\
\hline$a_{i}$ & 1731.5 & 4327.3 & 28321 & 29745 & 25436 \\
\hline$g_{i}$ & 5.1404 & 2.4565 & 1.7640 & 1.2065 & 0.4930 \\
\hline$\eta_{i}(d B)$ & 1.4506 & 3.4083 & 5.8115 & 8.5375 & 20.5763 \\
\hline$\Gamma_{i}(d B)$ & 3.8 & 7.1 & 9.3 & 11 & 14.8 \\
\hline
\end{tabular}

\section{Adaptive Modulation and Coding (AMC) scheme}

The objective of AMC is to maximize the system throughput by adjusting the transmission parameters like modulation order and coding rate according to the available channel state information (CSI), while maintaining the required packet error rate (PER). Usually, the effective SNR over the multiple subcarriers per user is used for MCS selection in generalized multi-carrier systems, which can be calculated as

$$
\gamma_{\text {eff }}= \begin{cases}-\beta \ln \frac{1}{M} \sum_{k=0}^{M-1} \exp \left\{-\frac{\gamma_{k}}{\beta}\right\}, & \text { OFDM } \\ \left\{\frac{1}{\frac{1}{M} \sum_{k=0}^{M-1} \frac{\gamma_{k}}{\gamma_{k}+1}}-1\right\}^{-1}, & \text { SC - FDMA }\end{cases}
$$

where $\gamma_{k}$ represents the instantaneous signal-to-noise ratio(SNR) at the $k$ th subcarrier, and $\beta$ is a parameter that must be optimized from link-level simulation results for each MCS by exponential effective signal-to-interference-noise ratio mapping (EESM) method [8].

Assume that there are $I$ modulation and coding schemes (MCSs) available for transmission in the systems. The entire received SNR region is partitioned into $I+1$ by region boundary denoted by $\left\{\Gamma_{i}\right\}_{i=0}^{I+1}$. The AMC is performed according to the received SNR as

$$
\text { Mode } i \text { is chosen when } \gamma_{\text {eff }} \in\left[\Gamma_{i}, \Gamma_{i+1}\right) \text {. }
$$

For simplicity, the PER performance of each MCS in the presence of additive white Gaussian noise (AWGN) can be approximatively expressed as [9]

$$
P_{i}\left\{\gamma_{e f f}\right\} \approx\left\{\begin{array}{l}
1, \text { if } 0 \leq \gamma_{e f f}<\eta_{i} \\
a_{i} \exp \left(-g_{i} \gamma_{e f f}\right), \text { if } \gamma_{e f f} \geq \eta_{i}
\end{array},\right.
$$

where the coefficients of $a_{i}, g_{i}, \eta_{i}$ are mode-dependent as shown in Table I. These coefficients are generated by fitting and comparing curves to the simulated PER according to the Monte-carlo simulations with parameters given by $3 \mathrm{G}$ LTE specification [10]. We first set $\Gamma_{0}=0$ and $\Gamma_{I+1}=\infty$. For various MCS modes on the assumption of target $P E R=10^{-2}$, the SNR boundaries can be found in Table I.

\section{Hybrid Multiple AcCess Scheme}

Since the OFDM is more sensible to the non-linearity of HPA than SC-FDMA, less signal power efficiency and more interference will be caused by the HPA with the higher transmission power in OFDM systems. Then, the switch from OFDM to SC-FDMA is necessary when these effects have deteriorated the performance of OFDM more seriously than that of SC-OFDM systems. Furthermore, when the effects of 
non-linearity become quite serious, the AMC can't achieve the performance gain because of inaccuracy of MCS selection. So it has to be disabled and turns to the non-adaptive transmission even in SC-FDMA systems. Therefore, we propose a hybrid multiple access method for uplink transmission, which includes three MA modes, i.e. MA1 (OFDM with AMC), MA2 (SC-FDMA with AMC) and MA3 (SC-FDMA with MCS1). Considering the effects of non-linearity, the MA mode can be switched according to the varying PER performances. In principle, such MA switch is happened every $W$ frames, which is not as frequently as MCS selection. The details of MA selection is described as following:

Step 1: In order to monitor the performance changes, the average PER is measured by checking CRC at the receiver, i.e.

$$
\xi=\frac{1}{W} \sum_{t=1}^{W} f(t)
$$

where the index $t$ represents the frame index within every $W$ frames, and $f(t)$ is the flag of the transmission status of the codeword $t$, i.e. $f(t)=0$ in case of correct transmission, otherwise, $f(t)=1$.

Step 2: On the assumption that one of MA modes is selected for transmission, the PER performance with ideal HPA is expected as

$$
\tilde{\xi}=\left\{\begin{array}{l}
\sum_{i=1}^{I} \frac{1}{W} \sum_{t=1, t \in \mathcal{M}_{i}}^{W} P_{i}\left\{\gamma_{e f f}(t)\right\}, \text { MA1/MA2 } \\
\frac{1}{W} \sum_{t=1}^{W} P_{1}\left\{\gamma_{e f f}(t)\right\}, \quad \text { MA3 }
\end{array}\right.
$$

where $\gamma_{e f f}(t)$ is the effective SNR in frame $t$ and can be calculated by (7), $\mathcal{M}_{i}=\left\{t \mid \gamma_{e f f}(t) \in\left[\Gamma_{i}, \Gamma_{i+1}\right)\right\}$ is the set that mode $i$ is selected for transmission in every $W$ frames.

Step 3: Next, let us compute the PER difference between the measured and expected PER as

$$
\varepsilon=|\xi-\tilde{\xi}|,
$$

with the initialization value of zero. It can reflect the nonlinearity effects clearly. More serious non-linear region the HPA works in, the larger $\varepsilon$ becomes. Therefore, based on this metric $\varepsilon$, the MA mode can be selected as shown in Fig.2. For example, on the assumption that MAI (OFDM with AMC) is adopted currently, the system will switch to MA2 (SC-FDMA with AMC) if the $\epsilon$ becomes no less than the threshold $\lambda_{1,2}$, i.e. $\varepsilon \geq \lambda_{1,2}$. Oppositely, it can change the MA mode from MA2 to MA1 in case of $\varepsilon<\lambda_{2,1}$. If the effects of nonlinearity becomes more seriously, i.e. $\varepsilon \geq \lambda_{2,3}$, the AMC will be disabled with SC-FDMA scheme, i.e. MA3(SC-AMC with MCS1). Correspondingly, when the $\varepsilon$ becomes less under the non-adaptive transmission, i.e. $\varepsilon<\lambda_{1,2}$, the system will try to enable the AMC again. The values of all the thresholds can be found by the simulations as explained in the next section.

Usually, the transmission power of the mobile station won't be adjusted very rapidly so that the changes of HPA characters are either. Therefore, the transition between different MAs are only happened between the adjacent MA modes, i.e. between $M A 1$ and MA2, between MA2 and MA3.

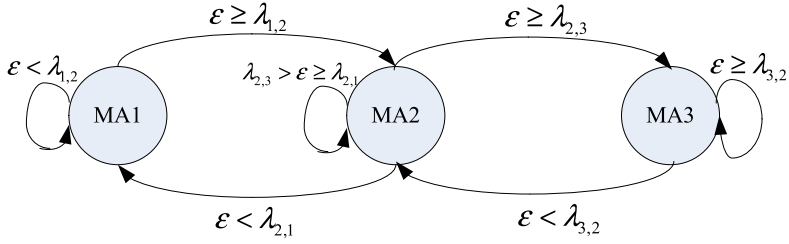

Fig. 2. Transitions between the adjacent MA modes.

TABLE II

SimUlation PARAMETERS

\begin{tabular}{c|c}
\hline Channel model & Pedestrian B \\
\hline Mobile Speed & $3 \mathrm{~km} /$ hour \\
\hline Path loss $(\mathrm{dB})$ & $128.1+37.6 \log _{10} d$ in $\mathrm{km}$ \\
\hline Carrier frequency $(\mathrm{GHz})$ & 2.0 \\
\hline Sample frequency $(\mathrm{MHz})$ & 15.36 \\
\hline Subcarrier interval $(\mathrm{kHz})$ & 15 \\
\hline Guard interval $($ samples $)$ & 73 \\
\hline DFT size $\left(N_{c}\right)$ & 1024 \\
\hline Frame length $(m s)$ & 0.5 \\
Number of subcarriers per user & 48 \\
\hline Noise power & $-12 \mathrm{~dB}$ \\
\hline Channel estimation & Least Square(LS) \\
\hline$p$ in Rapp model & 2 \\
\hline
\end{tabular}

Step 4: The decision of MA selection is made at the base station and feedback to the mobile user every $W$ frames.

\section{Simulation Results}

The impacts of amplifier nonlinearities on performances of systems with different multiple access methods are evaluated through simulations in this section, where the key system parameters are specified in Table II [10]. For simplicity, the ideal power control (PC) is assumed only to combat the the power loss due to path loss in our simulations. AMC and PC are carried out based on the averaged received SNR so as to obtain the throughput performance.

Fig. 3 compares the throughput of the systems in case of different MA modes. When the distance $d$ between the mobile user and base station is short, the signal gain of the amplifier at the terminal is so small that the non-linearity effects can be ignored. Then, the performance of OFDM systems is better than that of SC-FDMA systems because it can better exploit the channel frequency diversity gain with the help of the powerful channel coding. With the increase of the distance between the mobile user and base station, more serious distortion occurs due to higher transmission power so that less power efficiency and more interference are caused by the non-linearity of HPA especially in OFDM systems. It can be seen that the throughput performance of OFDM systems deteriorates more rapidly than that of SC-FDMA systems with more serious effects of non-linearity. So, when hybrid multiple access method is applied, the switch from MA1 to MA2 will 


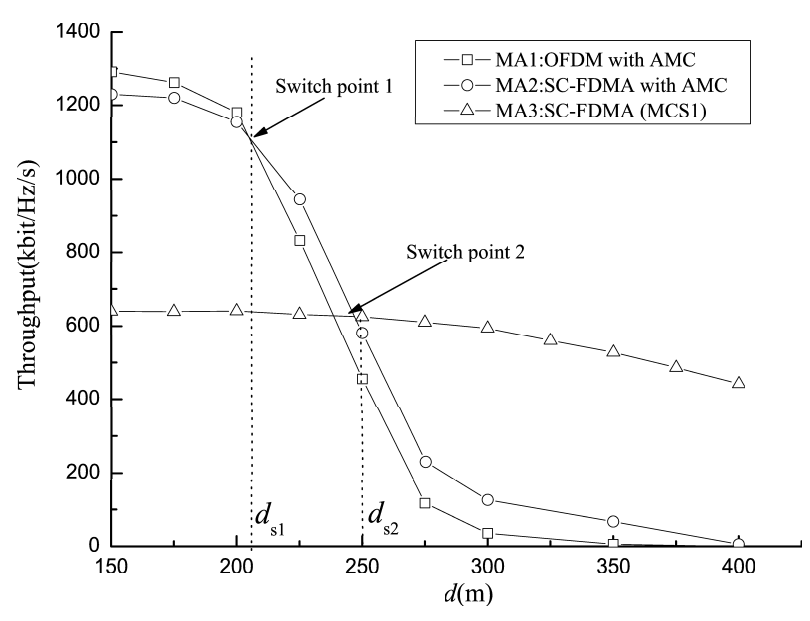

Fig. 3. Throughput comparison between different MAs.

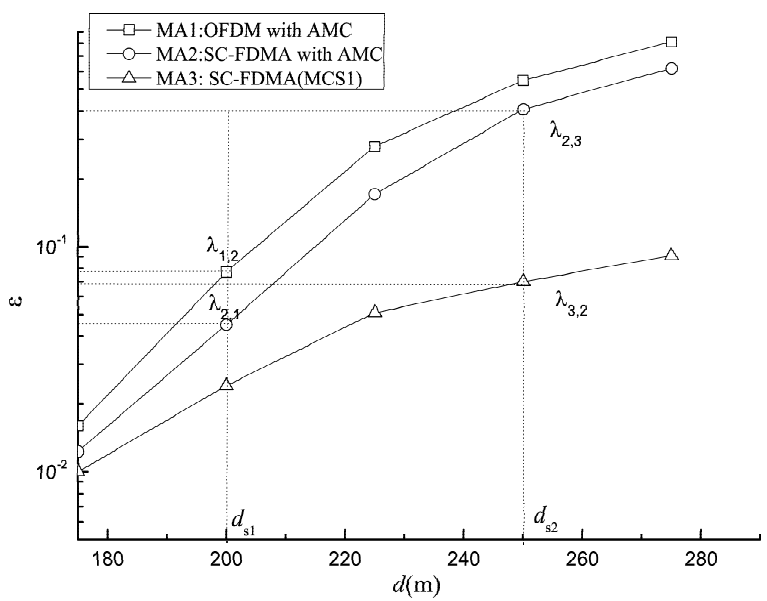

Fig. 4. $\varepsilon$ measurement with different MAs.

be happened when the throughput of the former is less than that of the latter, e.g. in case that the user distance is larger than $d_{s_{1}}=210 \mathrm{~m}$, and vice versa. When the transmission power of the user is further increased in case of the larger distance between the user and base station (e.g. larger than $d_{s_{2}}=250 \mathrm{~m}$ ), the measured SNR at the receiver becomes inaccurate due to serious non-linearity and AMC scheme can't work well. In this case, the AMC scheme chooses too higher MCS mode with wrong CSI so that most of transmissions are failed and low throughput happened. Therefore, now it is necessary to disable the AMC scheme and switch to MA3.

Next, let us find the threshold value for switching between different MA scheme by simulations with the average window of 5000 frame, i.e. $W=5000$. On the assumption that one of the possible MA modes including MA1, MA2 and MA3 is applied, Fig.4 illustrates the PER difference between the measured and expected PER, i.e. $\varepsilon$. Then, we can find the threshold value of the proposed hybrid multiple method according to the switch points shown in Fig.3. For example, corresponding to the first switch point at $d_{s 1}$, the threshold for switching from MAl to $M A 2$ can be set as $\lambda_{1,2}=0.078$. In the other words, when the base station detects that the PER difference $\varepsilon$ is larger than $\lambda_{1,2}=0.078$, it will then select MA2 instead of currently-used $M A 1$ as its multiple access method. Similarly, another kinds of thresholds, including from $M A 2$ to $M A 1$, from $M A 2$ to $M A 3$, and from $M A 3$ to $M A 2$, can also be found from Fig.4, i.e. $\lambda_{2,1}=0.046, \lambda_{2,3}=0.400, \lambda_{3,2}=0.069$.

\section{CONClusion}

In this paper, we present the framework of the uplink generalized multi-carrier systems while considering the effects of adaptive modulation and non-linear amplifier at the mobile user. It is shown that OFDM is superior to SC-FDMA when the user is close to the base station. On the other hand, the performances of SC-FDMA are better than those of OFDM in case that the user is around the cell edge because it is less sensitivity to non-linearity of HPA. The cross-point of performances between SC-FDMA and OFDM depends on several parameters such as MCS mode, noise power, HPA characters and so on. So, in order to combine the advantages of both OFDM and SC-FDMA schemes in different application scenarios, we propose a hybrid multiple access scheme and demonstrate its effectiveness by our simulations.

\section{ACKNOWLEDGMENT}

This work was supported in part by National Key Technology R\&D Program of China under Grant 2009ZX03005-00302 and Research Fund for the Doctoral Program of Higher Education under Grant 200800131023.

\section{REFERENCES}

[1] 3GPP TR 25.814 V7.1.0, "Physical layer aspects for evolved Universal Terrestrial Radio Access (UTRA) (Release 7),” Sep, 2006.

[2] 3GPP TR 36.814 V1.4.1, "Further Advancements for E-UTRA Physical Layer Aspects (Release 9)," Sep. 2009.

[3] E. Costa, M. Midrio, S. Pupolin, "Impact of amplifier nonlinearities on OFDM transmission system performance," IEEE Communications Letters, vol.3, no.2, pp.37-39, February, 1999.

[4] V.A. Bohara, Ho Ting See, "Analysis of OFDM Signals in Nonlinear High Power Amplifier with Memory," IEEE International Conference on Communications, 2008, pp.3653 - 3657, May 19-23, 2008.

[5] J. Tubbax, B. Come, and L. Van der Perre, "OFDM versus Single Carrier with Cyclic Prefix: a system-based comparison," IEEE Vehicular Technology Conference, 2001. VTC 2001 Fall, vol. 2, pp.1115-1119, Oct. 2001.

[6] K. Zheng, M. Wei, H. Long; Y. Liu, W. Wang, "Impacts of amplifier nonlinearities on uplink performance in 3G LTE systems," IEEE international Conference on Communications and Networking in China, 2009,ChinaCOM 2009, pp.1-5, Aug. 2009.

[7] C. Rapp, "Effects of HPA-nonlinearity on 4-DPSK-OFDM-signal for a digital sound broadcasting system, in Proc. 2nd European Conference on Satellite Communications, Liege, Belgium, Oct, 1991, ESA-SP-332.

[8] 3GPP2-C30-20030429-010, Ericsson, "Effective SNR mapping for modelling frame error rates in multiple-state channels," April, 2003.

[9] Q. Liu, S. Zhou, and G. B. Giannakis, "Cross-layer combining of adaptive modulation and coding with truncated ARQ over wireless links," IEEE Trans.Wireless Commun., vol. 2, no. 5, pp. 1746-1775, Sep, 2004.

[10] 3GPP TS 36.211 Evolved Universal Terrestrial Radio Access (E-UTRA); Physical channels and modulation. 